\title{
Single-file dynamics of colloids in circular channels: Time scales, scaling laws and their universality
}

\author{
Alejandro Villada-Balbuena $\odot,{ }^{1,2, *}$ Antonio Ortiz-Ambriz $\odot,{ }^{3,4,5}$ Pavel Castro-Villarreal $\odot,{ }^{6}$ Pietro Tierno $\odot,{ }^{3,4,5}$ \\ Ramón Castañeda-Priego $\odot,{ }^{7}$ and José Miguel Méndez-Alcaraz $\odot^{2, \dagger}$ \\ ${ }^{1}$ Condensed Matter Physics Laboratory, Heinrich Heine University, Universitätsstraße 1, 40225 Düsseldorf, Germany \\ ${ }^{2}$ Departamento de Física, Cinvestav, Avenida Instituto Politécnico Nacional 2508, Col. San Pedro Zacatenco, \\ Gustavo A. Madero, 07360 Ciudad de México, Mexico \\ ${ }^{3}$ Departament de Física de la Matèria Condensada, Universitat de Barcelona, 08028 Barcelona, Spain \\ ${ }^{4}$ Universitat de Barcelona Institute of Complex Systems (UBICS), Universitat de Barcelona, 08028 Barcelona, Spain \\ ${ }^{5}$ Institut de Nanociència i Nanotecnologia, Universitat de Barcelona, 08028 Barcelona, Spain \\ ${ }^{6}$ Facultad de Ciencias en Física y Matemáticas, Universidad Autónoma de Chiapas, Carretera Emiliano Zapata, \\ Km. 8, Rancho San Francisco, 29050 Tuxtla Gutiérrez, Chiapas, Mexico \\ ${ }^{7}$ División de Ciencias e Ingenierías, Campus León, Universidad de Guanajuato, Loma del Bosque 103, 37150 León, Guanajuato, Mexico
}

(Received 7 June 2021; accepted 20 August 2021; published 14 September 2021)

\begin{abstract}
In colloidal systems, Brownian motion emerges from the massive separation of time and length scales associated with characteristic dynamics of the solute and solvent constituents. This separation of scales produces several temporal regimes in the colloidal dynamics when combined with the effects of the interaction between the particles, confinement conditions, and state variables, such as density and temperature. Some examples are the short- and long-time regimes in two- and three-dimensional open systems and the diffusive and subdiffusive regimes observed in the single-file (SF) dynamics along a straight line. In this paper, we address the way in which a confining geometry induces new time scales. We report on the dynamics of interacting colloidal particles moving along a circle by combining a heuristic theoretical analysis of the involved scales, Brownian dynamics computer simulations, and video-microscopy experiments with paramagnetic colloids confined to lithographic circular channels subjected to an external magnetic field. The systems display four temporal regimes in the following order: one-dimensional free diffusion, SF subdiffusion, free-cluster rotational diffusion, and the expected saturation due to the confinement. We also report analytical expressions for the mean-square angular displacement and crossover times obtained from scaling arguments, which accurately reproduce both experiments and simulations. Our generic approach can be used to predict the long-time dynamics of many other confined physical systems.
\end{abstract}

DOI: 10.1103/PhysRevResearch.3.033246

\section{INTRODUCTION}

Diffusion is one of the most common mechanisms used by nature to dissipate equilibrium density fluctuations, where the Brownian motion of colloidal particles represents a fascinating case [1]. It exhibits a rich dynamical scenario in an extended time window due to the enormous separation of the characteristic time and length scales of solute and solvent constituents, combined with the effects of the interaction between the particles, the confinement conditions, and the state variables such as density and temperature [2,3]. The way a confining geometry affects the colloidal diffusion has received much attention in the last decade [4,5]. However, from the

\footnotetext{
*villadab@uni-duesseldorf.de

$\dagger$ jmendez@fis.cinvestav.mx
}

Published by the American Physical Society under the terms of the Creative Commons Attribution 4.0 International license. Further distribution of this work must maintain attribution to the author(s) and the published article's title, journal citation, and DOI. experimental side, these effects are hard to study since they cover eight or more orders of magnitude, from milliseconds to several days $[4,6,7]$. This feature makes it difficult to stabilize experimental setups over such a long time, and there are just few simulation results due to their high demand for computational power, which has held back theoretical developments as well.

The mean-square displacement [MSD or $W(t)$ in the following] of individual particles provides a good description of Brownian motion in systems where the possibility of particles finding a path among the other ones follows Gaussian diffusion $[2,3]$. It also helps to describe the single-file diffusion (SFD) of particles moving along a straight line without mutual passage [8], a problem which is highly relevant in many scientific fields including biophysics and materials science [7]. SFD of colloidal particles was directly observed experimentally using paramagnetic or charged colloidal particles moving along a circle $[9,10]$, which found them to freely diffuse until reaching a subdiffusive regime, like the case of open and infinite straight lines [8].

During the last few decades, intensive research has been done to understand the universal fingerprint of the SFD 
behavior $[W(t) \sim \sqrt{t}]$, where a plethora of theoretical explorations has been taken out for this purpose (see Ref. [7] for a review). However, since experiments and simulations have finite size, effects were reported when investigating confined systems characterized by a finite number of particles. Indeed, using a Bethe ansatz [11], an exact analytical expression for the tagged particle probability density function for hard-core interacting colloids in a finite box was found by Lizana and Ambjörnsson [12], revealing the existence of three temporal regimes: normal diffusion, SFD, and a saturation regime, which we define here as the geometric regime (GR). Particles interacting with a screened electrostatic potential confined in a narrow box were found to exhibit good agreement with the expected scaling of SFD at intermediate times, whereas the GR was found at large times [13]. Additionally, the asymptotic behavior of the MSD was found to be $D_{N} t$, where $D_{N}=D_{0} / N$ for a finite system of $N$ colloids restricted to the line; due to this characteristic, collective behavior emerges, where the system behaves as a single particle with an effective mass $\mathrm{Nm}$, with $D_{0}$ and $m$ being the free-particle diffusion coefficient and the mass of each colloid [14], respectively.

All the above mechanisms have one common feature: they are caused by a geometrical and/or topological restriction. The tagged particle, as well as all interacting particles, experiences the effects of the geometry or topology imposed by the environment. The geometry can be manifested in various forms as the shape of the confinement (e.g., the parabolic confinement), the bounded domain of the narrow channel, the periodic structure of the substrate, the multilayer structure, or the circular channel, among the many other forms one can find in nature. Geometry and topology, combined with the nature of the interaction between the particles, produce diverse types of effects [15]. For instance, topological defects, as kinks and antikinks, emerge when a colloidal monolayer is driven across commensurate and incommensurate substrate potentials [16], as well as in highly dense systems of repulsive colloids in a narrow and periodic channel [17]. In some situations, curvature effects arise in the free diffusion processes over curved manifolds [18], where curvature becomes a fundamental physical quantity that acts just as an external field would on the particles.

Recently, the original Ermak-McCammon (EM) algorithm for Brownian Dynamics (BD) was extended to study Brownian motion of interacting particles confined on curved manifolds [4]. Particularly, the diffusion of a tagged particle without hydrodynamic interactions can be approached using the overdamped many-particle Langevin equation on an arbitrary plane curved file as follows [4]:

$$
\zeta \frac{d s_{i}}{d t}=\sum_{i \neq j} F_{i j}^{T}+\eta_{i},
$$

where $\zeta$ is the friction coefficient, the subindexes labeled the particles, $s_{i}$ is the arc-length displacement, and $F_{i j}^{T}$ and $\eta_{i}$ are the interparticle force and the stochastic force projected along the tangent direction at the $i$ th particle position, respectively. The stochastic force satisfies the fluctuation-dissipation theorem (see details in Ref. [4]). As a consequence of the tangent projection, all the dynamics occurs intrinsically along the curved file. In this case, the geometry encodes strong non- linear effects coupled to the interactions through the tangent projection. Indeed, paramagnetic colloids distributed along an ellipse were studied using Eq. (1), showing that curvature gradients induce inhomogeneities in the distribution of the particles along the file and providing evidence of metastable states through the behavior of the self-diffusion [5]. Furthermore, the preliminary example of paramagnetic particles confined in a circular channel studied previously [4] provided evidence of two temporal regimes beyond the subdiffusive one not seen in experiments [9,10] nor even in theoretical approaches for straight lines [8]. Thus, it becomes evident that the colloid dynamics in a ring features a richer dynamical scenario that has not been studied in detail and will allow us to understand the role of the geometry on the dissipation of the equilibrium density fluctuations, a topic that has been overlooked so far.

Thus, by combining video-microscopy experiments performed with paramagnetic colloidal particles confined to lithographic circular microchannels subjected to an external magnetic field with the EM algorithm for curved manifolds implemented for the paramagnetic colloids confined to a circle, we unravel the rich dynamical behavior of interacting colloidal systems that emerges due to the geometric confinement. Particularly, we focus on the angular distribution function and the mean-square angular displacement (MSAD) for several sets of the system parameters, namely, the number of particles $N$, the radius of the circle $R$, and the strength of the repulsive interaction between colloids $\Gamma$. We find that the colloidal dynamics displays a universal behavior characterized by the following four temporal regimes: (1) free diffusion, (2) SFD, (3) free-cluster diffusion, and (4) geometrical diffusion. We provide accurate analytical expressions for the crossover times between these temporal regimes, which are obtained from scaling arguments, and derive accurate analytical representations for the MSAD in terms of the crossover times.

In Sec. II, we present the experimental setups. In Sec. III, we describe the implemented EM algorithm for curved manifolds. In Sec. IV, some experimental and simulation results for the structure and MSAD are shown. Sections V and VI are dedicated to the time scales and scaling laws, respectively. In Sec. VII, we report precise analytical expressions for the MSAD. Finally, in Sec. VIII are some concluding remarks.

\section{EXPERIMENTAL SETUP}

We performed video-microscopy experiments with paramagnetic colloidal particles confined to lithographic microgrooves in an external magnetic field following the procedure outlined in Ref. [19] with an improved protocol that will be discussed in the next paragraphs to ensure stability for longer periods of time. The experimental results obtained allow us to report the four temporal regimes and related time scales predicted with BD simulations.

Circular channels were first drawn on a chromium (Cr) mask using direct write laser lithography $(\lambda=$ $405 \mathrm{~nm}, 5-7 \mathrm{~mm}^{2} \mathrm{~min}^{-1}$, DWL 66, Heidelberg Instruments Mikrotechnik $\mathrm{GmbH})$. A thin coverglass $(\sim 120 \mu \mathrm{m})$ was coated with a $2.8 \mu \mathrm{m}$ layer of AZ-1512HS photoresist (Microchem, Newton, MA) by spinning it at $1000 \mathrm{rpm}$ for $30 \mathrm{~s}$ and subsequently curing it at $95^{\circ} \mathrm{C}$ for $3 \mathrm{~min}$. To improve 
adhesion, before the photoresist, the glass surface was coated by a thin layer of TI Primer by spin coating for $20 \mathrm{~s}$ at $4000 \mathrm{rpm}$ and baking for $2 \mathrm{~min}$ at $120^{\circ} \mathrm{C}$. The photoresist was irradiated with ultraviolet (UV) light through the Cr mask for $3.4 \mathrm{~s}$ at a power of $21 \mathrm{~mW} \mathrm{~cm}{ }^{-2}$ (UV-NIL, SUSS Microtech) and then developed by submerging it for $45 \mathrm{~s}$ in a solution of 1 part of AZ400K developer in 4 parts water before washing with water.

A colloidal suspension was prepared by mixing $1 \mu \mathrm{L}$ of superparamagnetic particles of diameter $\sigma=2.8 \mu \mathrm{m}$ (Dynabeads M-270, which consist of a polymer matrix embedded with iron-oxide nanoparticles) with $1 \mathrm{~mL}$ of a $7 \mu \mathrm{M}$ solution of tetramethylammonium hydroxide (TMAH) in ultrapure water (Synergy UV-3, Millipore) at a $\mathrm{pH}$ of 7.2. TMAH allowed us to avoid the absorption of $\mathrm{CO}_{2}$ in water, which produces carbonic acid that glues the particles to the surfaces; this simple procedure enhanced the stability of the setup. A sample was prepared by sandwiching a droplet of solution between the structures and a top coverslip, separated and sealed with silicone vacuum grease (Dow Corning). The thickness of the sample was $\sim 100 \mu \mathrm{m}$. Before placing the top coverslip, the open sample was sonicated for 3 min to get rid of bubbles created by the superhydrophobicity induced by the concentric channels. The seal of the sample was essential to maintain the chemical stability of particles during the long experiments required to observe the different diffusion regimes. We have observed that a properly sealed sample is stable for $\sim 2$ weeks, before the dispersing medium becomes too acidic and electrostatic charges no longer are able to stabilize the particles over the substrate. During these long times, the sample is shielded from UV light to prevent further exposure of the photoresist. Particles have a density of around twice that of water $\left(\approx 2 \mathrm{~g} \mathrm{~mL}^{-1}\right)$, which is enough to keep them inside the channels. The particles were suspended by electrostatic interactions a few hundred nanometers above the surface.

Once a sample was prepared, it was placed in a custommade optical microscope equipped with a $100 \times$ oil immersion objective (Nikon Plan Fluor, numerical aperture $=1.3$, used with Thorlabs MOIL-30, $n=1.518$ ), a laser for optical trapping (ML5-CW-P-TKS-OTS, Manlight, 5W, operated at 3W), and a coil to apply a field along the axial direction (see Fig. 1). The optical tweezers were used to move particles between the ring microchannels. The laser was deflected by an Acousto Optic Device (AOD AA Optoelectronics DTSXY-400-1064, driven by a radiofrequency wave generator DDSPA2X-D431b-34 and a NI cDAQ card NI-9403), which allowed fine control over the trap position and power. The time sharing of laser light is especially important since magnetic particles absorb light at this wavelength, and they are prone to heating. We addressed this by keeping the power coming into the microscope objective between 1 and $2 \mathrm{~mW}$. To have the power approximately constant when moving the trap position, a telescope conjugated the plane of the AOD to the back focal plane of the objective. The system was operated by a custom-made graphical interface programed in LABVIEW [20]. Observation was then done using a complementary metal oxide semiconductor camera (Ximea MQ003MG-CM, $640 \times 480$ pixels, pixel size was $7.4 \mu \mathrm{m}$ ) working at a rate of $15 \mathrm{fps}$ for short time scales and $1 \mathrm{fps}$ for long time scales. From the videos, the particle positions were extracted using
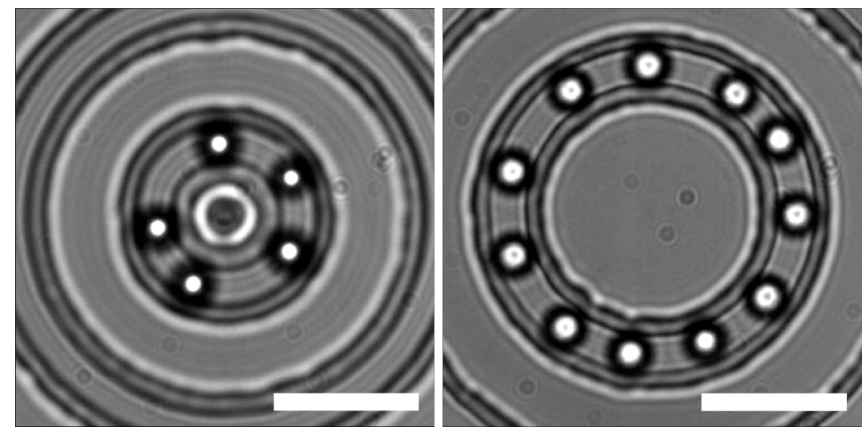

FIG. 1. Microscope image of colloidal particles confined by gravity in a microchannel. Left: Channel radius $R=5 \mu \mathrm{m}$ and 5 particles. Right: Channel radius $R=10 \mu \mathrm{m}$ and 11 particles. The widths of the channels are $2.5 \mu \mathrm{m}$ at the bottom and $5 \mu \mathrm{m}$ at the top, with a depth of $2.8 \mu \mathrm{m}$. The channel radius is measured from the center of the channel. Scale bar is $10 \mu \mathrm{m}$ in both images.

the TRACKPY implementation of the Crocker-Grier algorithm [21].

\section{BROWNIAN DYNAMICS SIMULATIONS ON THE CIRCLE AND SUPERPARAMAGNETIC POTENTIAL}

We used the EM algorithm over curved manifolds developed in Ref. [4] to perform a systematical analysis of the interacting diffusing particles on the circle $S^{1}$.

According to Ref. [4], the many-particle Langevin Eq. (1) on $S^{1}$ without hydrodynamic interactions can be rewritten as the following stochastic finite differences equation:

$$
s_{i}(t+\Delta t)=s_{i}(t)+\beta D_{0} \mathbf{F}_{i} \cdot \mathbf{T}_{i} \Delta t+\delta \mathbf{r}_{i}(\Delta t) \cdot \mathbf{T}_{i},
$$

where $s_{i}(t)$ is the arc length of the circle that can be computed using $s_{i}(t)=R \phi_{i}(t)$, with $\phi_{i}(t)$ being the angle measured counterclockwise from the positive $x$ axis in the Cartesian plane at time $t$. The total force $\mathbf{F}_{i}=\sum_{i \neq j=1}^{N} \mathbf{F}_{i j}$ exerted on the $i$ th particle is the sum of the forces $\mathbf{F}_{i j}$ due to the interactions with all the particles $j \neq i$. In Eq. (2), $\delta \mathbf{r}_{i}(\Delta t)$ is a Gaussian random displacement in the plane containing the circle with zero mean $\left\langle\delta r_{i, \alpha}(\Delta t)\right\rangle=0$ and covariance ma$\operatorname{trix}\left\langle\delta r_{i, \alpha}(\Delta t) \delta r_{j, \beta}(\Delta t)\right\rangle=2 D_{0} \delta_{i j} \delta_{\alpha \beta} \Delta t$, with $\delta r_{i, \alpha}(\Delta t)(\alpha=$ $1,2)$ and $\langle\cdots\rangle$ denoting, respectively, the Cartesian components of $\delta \mathbf{r}_{i}(\Delta t)$ and the ensemble averages. Here, $\beta=$ $\left(k_{B} T\right)^{-1}$ is the inverse of the thermal energy $k_{B} T$, with $k_{B}$ and $T$ being the Boltzmann's constant and the absolute temperature, respectively. Additionally, the unit tangent vector $\mathbf{T}_{i}=\left[-\sin \phi_{i}(t), \cos \phi_{i}(t)\right]$ appearing in Eq. (2) reflects the fact that the short-time dynamics occurs along the tangent line at the angle $\phi_{i}(t)$. According to those definitions, the arclength displacement $\Delta s_{i}(\Delta t)=s_{i}(t+\Delta t)-s_{i}(t)$ is related to the angular displacement $\Delta \phi_{i}(\Delta t)=\phi_{i}(t+\Delta t)-\phi_{i}(t)$ by $\Delta s_{i}(\Delta t)=R \Delta \phi_{i}(\Delta t)$; hence, the angular displacement is the sum between the deterministic part $\beta D_{0} \mathbf{F}_{i} \cdot \mathbf{T}_{i}$ and the random term $\delta \mathbf{r}_{i}(\Delta t) \cdot \mathbf{T}_{i}$.

In both experiments and simulations, we studied a two-dimensional colloidal dispersion composed of $N$ paramagnetic particles with a magnetic moment $M$, whose pair 
potential is given by [22]

$$
\beta u\left(r_{i j}\right)=\frac{\mu_{0}}{4 \pi k_{B} T} \frac{M^{2}}{r_{i j}^{3}}=\frac{\Gamma}{r_{i j}^{* 3}},
$$

where $\mu_{0}$ is the vacuum magnetic permeability. For weak magnetic fields $M(B)=\chi_{\text {eff }} B$ holds, with $\chi_{\text {eff }}$ being the effective magnetic susceptibility of the colloids and $B$ the external magnetic field [22]. The experiments were carried out at room temperature $(T=300 \mathrm{~K})$ with a magnetic susceptibility of $\chi_{\text {eff }}=(0.366 \pm 0.0002) \times 10^{-11} \mathrm{Am}^{2} / \mathrm{T}$. The right-hand side term in Eq. (3) is the resulting dimensionless interaction potential, where $\Gamma$ is the total amplitude, and $r_{i j}^{*}=r_{i j} / \sigma$ is the Euclidean distance between particles, expressed in terms of the particle diameter $\sigma$. As a result of the confinement and the finite size of the particles, the Euclidean distance $r_{i j}=\sqrt{2} R \sqrt{1-\cos \left(\phi_{i}-\phi_{j}\right)}$ is bounded from above and below with maximum value $r_{i j}=2 R$ when $\phi_{i}-\phi_{j}= \pm \pi$ and minimum value $r_{i j}=\sigma$ when $\phi_{i}-\phi_{j}= \pm 2 \sin ^{-1}(\sigma / 2 R)$, respectively. Since the maximum angle between two particles is $\pi$, the domain of the angular distribution function $g(\phi)$ is $[0, \pi]$. By using this feature, for instance, previous results $[23,24]$ can be recovered for both dynamic and static properties.

BD simulations were carried out as follows. Here, $N$ colloidal particles were set in random initial positions along the circle. Then the colloidal system evolved according to Eq. (2) from its arbitrary initial nonequilibrium state to the equilibrium one when the energy of the system reached an average constant value, where the system was considered to be in equilibrium. The chosen time step for all the simulations was $\Delta t^{*}=\Delta t D_{0} / \sigma^{2}=10^{-5}$. After reaching thermal equilibrium in $10^{6}$ time steps, we used at least $5 \times 10^{11}$ time steps to gather statistics, taking configurations every 100 time steps. To get good statistics at long times, we parallelized the BD code to run 320 simulations of the same system, using a different time seed in each case and averaging over all of them when simulations are done.

\section{STRUCTURE AND MSAD: EXPERIMENTS VS COMPUTER SIMULATIONS}

To perform a one-to-one comparison between BD simulations and experiments, we chose four sets of experimental parameters, namely, $\{N=5, R=5 \mu \mathrm{m}, \Gamma=1.07\},\{N=$ $5, R=5 \mu \mathrm{m}, \Gamma=4.26\},\{N=5, R=5 \mu \mathrm{m}, \Gamma=9.59\}$, and $\{N=11, R=10 \mu \mathrm{m}, \Gamma=23.78\}$. In each case, $\Gamma$ was determined using the experimental parameters provided in the previous section. Also, different values for the magnitude of the magnetic field $\{B[\mathrm{mT}]=0.27,0.54,0.8,1.27\}$ were used in each set.

The angular distribution function $g(\phi)$, i.e., the probability of finding a particle forming an angle $\phi$ with another particle, is explicitly shown in Fig. 2. One can immediately notice that, for all four sets of parameters, there is an excellent agreement between experiments and simulations without using any free parameter, thus highlighting the accuracy of the modified EM algorithm in Eq. (2) to reproduce the trajectory of interacting colloids on curved manifolds. Furthermore, as expected, we also observe that $g(\phi)$ becomes highly structured and longrange correlated when $\Gamma$ and $N$ increase. When $\Gamma=1.07$, the

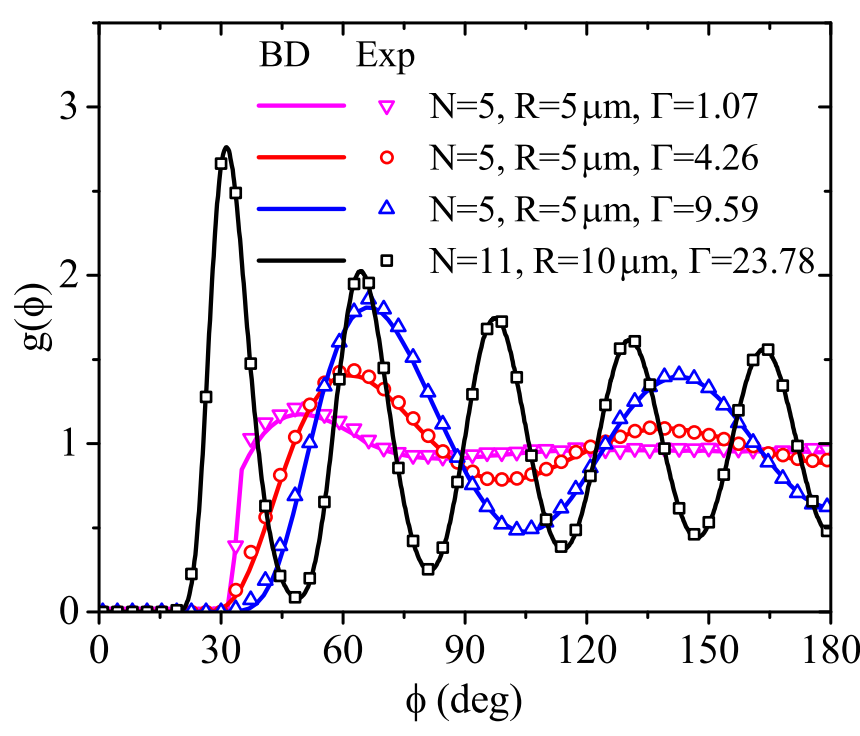

FIG. 2. Angular distribution function $g(\phi)$ for paramagnetic particles diffusing along a circle measured from video-microscopy experiments (open symbols) and calculated using Brownian dynamics (BD) simulations (solid lines) with the Ermak-McCammon (EM) algorithm for curved manifolds in Eq. (2) for four sets of system parameters, as indicated.

paramagnetic repulsion is not enough to prevent the particles from colliding, as seen from the breaking of the pink line when reaching the correlation hole. We do consider volume exclusion in our simulations, but the effect is so slight that it does not have implications for the diffusion of the particles.

In addition, in Fig. 3, the MSAD $\left\langle[\Delta \phi(t)]^{2}\right\rangle$ is shown for the same set of system parameters as the one used for the calculations of the $g(\phi)$ displayed in Fig. 2. Just as in a previous contribution [4], we note that $\left\langle[\Delta \phi(t)]^{2}\right\rangle$ exhibits systematically the following four temporal regimes: the shorttime diffusive regime (I), the subdiffusive intermediate time regime (II), the second diffusive regime (III), and finally, the geometrical saturation regime (IV). From the figure, we also note an excellent agreement between experiments and BD simulations at short and first intermediate times; however, at the second intermediate time regime, one can notice small differences that slightly increase with $\Gamma$. Overall, the agreement is outstanding given that there are no free parameters in the calculations. Afterwards, at the geometrical regime, a larger difference is evident for $N=11$ as a consequence of a lack of statistics in the experiment, mainly because of the difficulty to keep the measurement stable for very long times.

\section{TIME SCALES FROM THE MSAD}

Given the excellent agreement between experiments and Brownian dynamics simulation results discussed previously, we now proceed to explore additional sets of the system parameters fixing in each case two of the following three physical variables $N, R^{*}=R / \sigma, \Gamma$ and varying the remaining one:

(1) $\left\{R^{*}=10.5 ; \Gamma=125 ; N=1,10,15,20,25,32\right\}$,

(2) $\left\{N=26 ; \Gamma=125, R^{*}=10.5,13,15.5,18,20.5,30.5\right\}$, and 


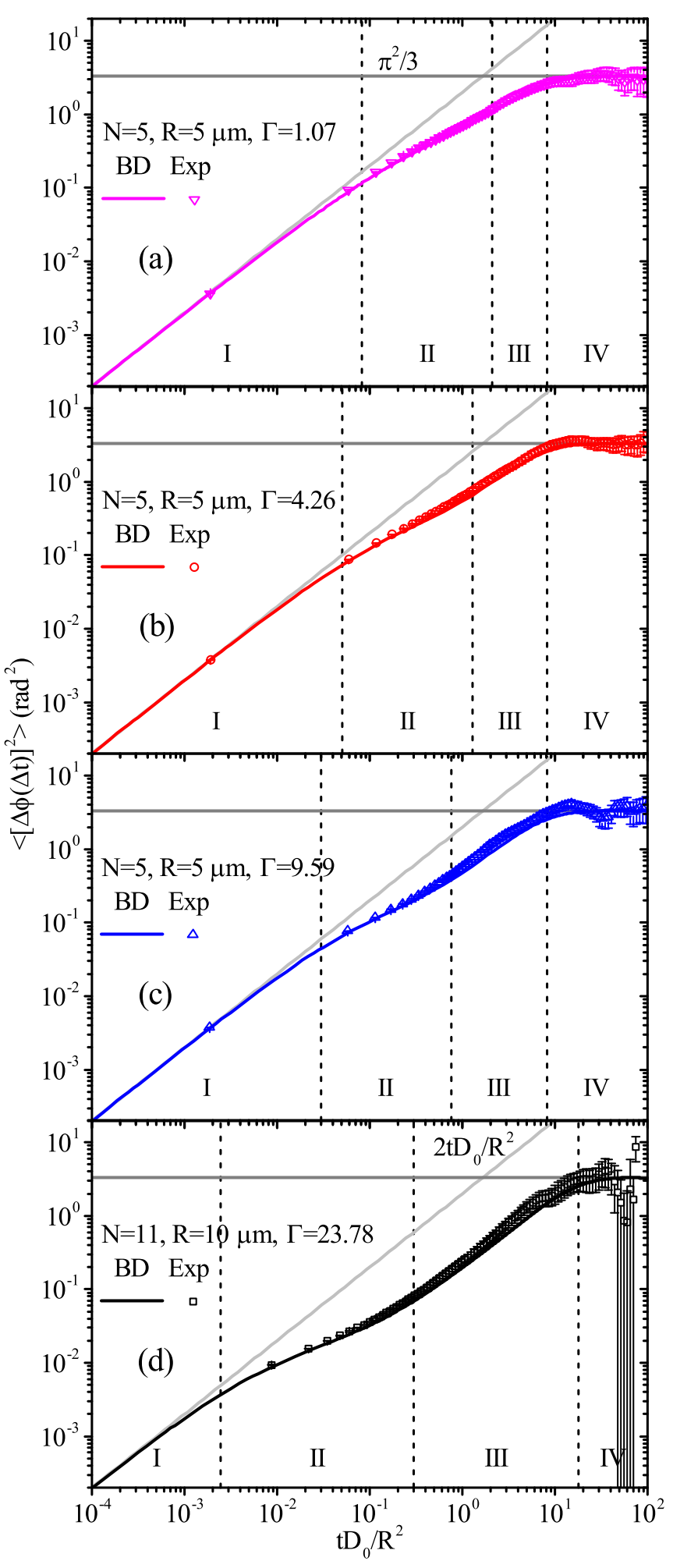

FIG. 3. Mean-square angular displacement $\left\langle[\Delta \phi(t)]^{2}\right\rangle$ from experiments (open symbols) and Brownian dynamics (BD) simulations (solid lines). For all sets of curves, we identify the following four temporal regimes: the short-time diffusive regime (I), the subdiffusive intermediate time regime (II), the second diffusive regime (III), and the geometrical saturation regime (IV). The straight gray lines are guides for the eye.
(3) $\left\{N=10 ; R^{*}=10.5 ; \Gamma=10,10^{2}, 10^{3}, 10^{4}\right\}$.

Results for the MSAD are shown in Fig. 4. It turns out that the four time regimes depicted in Fig. 3 are also observed in the dynamical behavior of the MSAD of Fig. 4. To better understand the dynamical behavior at each regime, we will now classify all of them in terms of the characteristic transition times $\tau_{i}$ that define their beginning and end: short-time regime $t \leqslant \tau_{d}$, first intermediate time regime $\tau_{d} \leqslant t \leqslant \tau_{c}$, second intermediate time regime $\tau_{c} \leqslant t \leqslant \tau_{G}$, and geometrical regime $t>\tau_{G}$, where $\tau_{d}, \tau_{c}$, and $\tau_{G}$ depend clearly on the physical parameters $N, R^{*}$, and $\Gamma$. One of the main goals of this contribution is to express the dependence of these transition times on the aforementioned physical quantities. We now explain the behavior of the MSAD in each of these regimes.

According to the results shown in Fig. 4, at the short-time regime, the MSAD displays a one-dimensional (1D) freeparticle-like behavior:

$$
\left\langle[\Delta \phi(t)]^{2}\right\rangle=2 \frac{D_{0}}{R^{2}} t .
$$

This behavior states that, at short times, the deterministic term $\beta D_{0} \mathbf{F}_{i} \cdot \mathbf{T}_{i} \Delta t$ is negligible in comparison with the random term $\delta \mathbf{r}_{i}(\Delta t) \cdot \mathbf{T}_{i}$ in the EM algorithm in Eq. (2). This implies that, at short times, fluctuations play an essential role in contrast to the interactions between the particles. Also, a 1D Brownian motion is found since the circle is a 1D manifold, which can be considered locally as a straight line.

In the first intermediate regime, the interactions between the particles take place, and this results in a deviation of the MSAD from the free-particle behavior to the dynamical transition known as SFD:

$$
\left\langle[\Delta \phi(t)]^{2}\right\rangle=\frac{F \sqrt{t}}{R^{2}},
$$

where $F$ is the SF mobility, and the dependence $\sqrt{t}$ is inferred from the slope of the curve in Fig. 4. SFD occurs because of the excluded mutual passage between the particles [7]. Consequently, this phenomenon appears also for any type of repulsive interaction, even for the simplest hard-core ones, if the particles are kept confined in the single line [7]. Recall that the transition to the SFD occurs sooner as the particle density increases, which is achieved when either $N$ increases or $R$ decreases. According to the prediction reported in Ref. [8], the coefficient $F$ depends on the relative compressibility. In our case, we will show below how $F$ depends on the strength of the interaction potential.

In the third regime, the curves of the MSAD are basically parallel to the curve of the free-particle behavior, which indicates that the MSAD is again proportional to time $t$. In addition, it turns out that the proportionality coefficient is $2 D_{0} / N$. Thus, the dynamical behavior at the second intermediate time regime can be expressed as follows:

$$
\left\langle[\Delta \phi(t)]^{2}\right\rangle=2 \frac{D_{0}}{N R^{2}} t .
$$

Noticeably, this result highlights the fact that the whole bunch of particles confined in the circle behaves as if the system were 


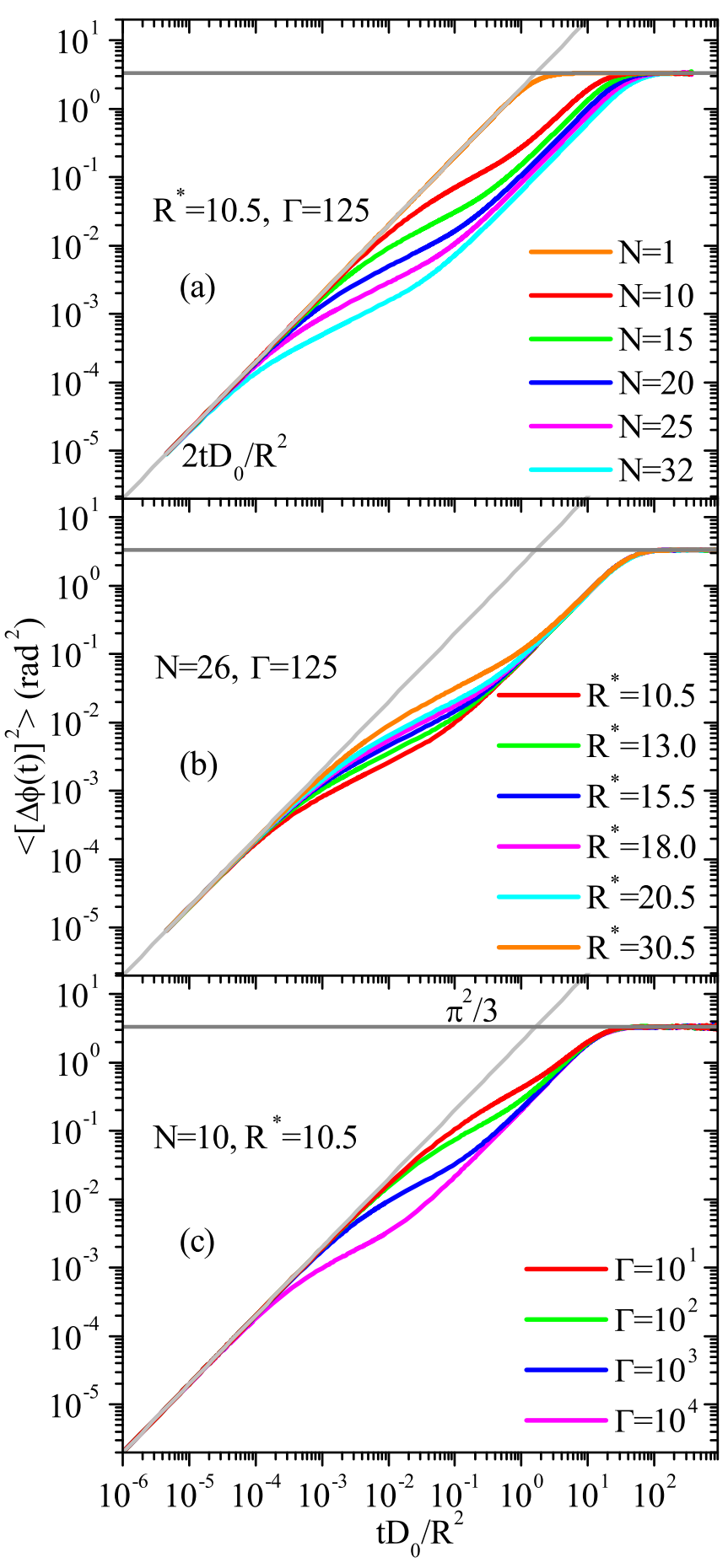

FIG. 4. Mean-square angular displacement $\left\langle[\Delta \phi(t)]^{2}\right\rangle$ obtained with the Ermak-McCammon (EM) algorithm in Eq. (3) for paramagnetic colloids diffusing along a circle (a) for different numbers of particles, (b) varying the radius of the circle, and (c) changing the strength of the interparticle repulsion, as indicated. The thin straight lines are guides for the eyes, showing the short-time $2 D_{0} t / R^{2}$ and the geometrical limit $\pi^{2} / 3$ values.

just one particle in a fluid with an effective drag coefficient $\zeta_{N}=N \zeta$, where $\zeta=k_{B} T / D_{0}$ is the drag coefficient between any of the colloids and the fluid. As a result, at this time regime, a collective behavior emerges where the whole system acts as one ring-shaped particle with an effective diffusion coefficient $D_{N}=D_{0} / N$ performing a rotational Brownian motion. In other words, the particles organize in such a way that they randomly move together as a cluster, and again, the collective dynamics is independent from the interaction potential. This phenomenon is a consequence of the finite size of the system imposed by the topology of the circle, and it does not have a counterpart for the case of open systems. A similar situation appears in the case of the finite-sized colloidal system confined in a line [14]. We expect this kind of transition to be universally valid for any repulsive interaction potential if the particles are constrained along a circle [25]. In other words, we find a transition from the SFD to the cluster file diffusion.

Finally, in the fourth time regime, the system reaches the so-called GR, where particles have explored the positions of the circle many times in such a way that any colloid has the same probability to be found at any position on the circle, independently of the interaction between the particles. This result was previously reported in Ref. [4] and has an analytical value:

$$
\left\langle[\Delta \phi(t)]^{2}\right\rangle=\frac{\pi^{2}}{3} .
$$

Thus, from the previous discussion and the dynamical behavior reported in Fig. 4, one can appreciate that the only parameters undetermined are the SF mobility $F$ and the transition times $\tau_{d}, \tau_{c}$, and $\tau_{G}$ that we determine in the following.

\section{CROSSOVER TIMES: SCALING LAWS}

We now provide a deduction of the SF mobility and the transition times $\tau_{d}, \tau_{c}$, and $\tau_{G}$. First, let us notice that, from the EM algorithm in Eq. (2), we can show that

$$
\left\langle\left[\Delta s_{i}(\tau)\right]^{2}\right\rangle=\left\langle\left[\beta D_{0} \tau \mathbf{F}_{i} \cdot \mathbf{T}_{i}\right]^{2}\right\rangle+2 D_{0} \tau,
$$

by squaring Eq. (2) and taking its average. The crossed product does not appear in Eq. (8) since the deterministic term $\beta D_{0} \mathbf{F}_{i} \cdot \mathbf{T}_{i} \tau$ and the tangent vector $\mathbf{T}_{i}$ in the random term both depend on the previous time $t$ with respect to $t+\tau$. Clearly, when interactions are absent, the last equation reproduces the behavior of the MSAD at the short-time regime in Eq. (4). In addition, when interparticle interactions are considered, the first term of Eq. (8) becomes important. Indeed, this term has the same order of magnitude of the second one when the system reaches the SFD regime.

To determine the first transition time, first, let us carry out a dimensional analysis, where we denote by $[\mathcal{Q}]$ the dimension of the quantity $\mathcal{Q}$. Hence, the deterministic term $\beta D_{0} \mathbf{F}_{i} \cdot \mathbf{T}_{i} \tau$ in the EM algorithm in Eq. (2) has dimension of length. Thus, let us denote by $\ell$ a characteristic length to be inferred from the simulation. Then $\ell=\beta D_{0}\left(\mathbf{F}_{i} \cdot \mathbf{T}_{i}\right) \tau$, where $\tau$ is a characteristic time associated with $\ell$. The tangent vector $\mathbf{T}_{i}$ does not have any dimensions; thus, $\left(\mathbf{F}_{i} \cdot \mathbf{T}_{i}\right)=\mathcal{U} / \ell$, where $\mathcal{U}$ has units of energy. Combining all these terms, one gets the following expression:

$$
\frac{D_{0} \tau}{\sigma^{2}}=\mathcal{C}\left(\frac{\ell}{\sigma}\right)^{2}(\beta \mathcal{U})^{-1} .
$$

In this equation, there are three quantities to be determined, namely, the characteristic length $\ell$, the value of $\mathcal{U}$, and the dimensionless constant $\mathcal{C}$. As it was explained earlier, the 
interaction term becomes relevant at the intermediate time regime; therefore, the first and second transition times should have the form shown in Eq. (9). The transition to SF behavior occurs when a particle approaches another one, such that the interaction becomes important and prevents mutual passage; thus, this should occur at the angle $\phi_{\max }$, which corresponds to the first peak of $g(\phi)$ that gives the maximum probability that two particles encounter each other. Additionally, it is known that $g(\phi)$ carries information of the interaction potential. Therefore, one could infer that, for the transition times in Eq. (9), $\mathcal{U}=u\left(r_{\max }\right)$, where $r_{\max }=\sqrt{2} R\left(1-\cos \phi_{\max }\right)^{1 / 2}$. Now to determine the value of $\ell$, we have performed perturbation theory on the EM algorithm in Eq. (2) and found that, for the first transition time, it has the form $D_{0} \tau_{d} / \sigma^{2}=$ $\mathcal{C}_{\text {th }}\left(R^{*} / N\right)^{2}\left[\beta u\left(r_{\text {max }}\right)\right]^{-1}$, where the theoretical value for the dimensionless constant is $\mathcal{C}_{\text {th }} \approx 1.3$ (see the Appendix). From this analysis, it turns out that $\ell=R / N$, and according to the simulation result displayed in Fig. $5, \mathcal{C} \approx 1$. Thus, one has the following mathematical expression for $\tau_{d}$ :

$$
\frac{D_{0} \tau_{d}}{\sigma^{2}}=\left(\frac{R^{*}}{N}\right)^{2}\left[\beta u\left(r_{\max }\right)\right]^{-1} .
$$

This expression has an excellent agreement with both experimental and simulation results, as it is shown on the top plot of Fig. 5.

The deduction of the SF mobility $F$ can be carried out using $\tau_{d}$, Eqs. (4) and (5). This can be obtained by equating Eq. (4) with the free-particle diffusion and Eq. (5) with the SFD at the first transition time $\tau_{d}$, namely, $2 D_{0} \tau_{d}=F \sqrt{\tau_{d}}$, that is,

$$
F=\left(\frac{2 R}{N}\right) \sqrt{\frac{D_{0}}{\beta u\left(r_{\max }\right)}} .
$$

This expression is straightforward and reproduces correctly both the experiments and simulations [see Figs. 5(a) and 6]. In fact, from Fig. 6, one can see the remarkably good agreement between the expressions in Eqs. (5) and (11) when compared with the simulation results. As pointed out above, Kollmann [8] derived an expression for $F$ in terms of the compressibility or the evaluation of the structure factor at $q \rightarrow 0$. However, in practice, that route needs the simulation of larger system sizes and, consequently, demands a high computational cost, thus making Eq. (11) more feasible to explain the transport of particles when the SFD condition is reached.

Now using Eq. (5) with the value for $F$ given by Eq. (11), one can proceed to deduce the second transition time $\tau_{c}$ by equating Eqs. (5) and (6). Hence, one now obtains that

$$
\frac{D_{0} \tau_{c}}{\sigma^{2}}=R^{* 2}\left[\beta u\left(r_{\max }\right)\right]^{-1} .
$$

As it can also be seen in Fig. 5(b), the previous expression for $\tau_{c}$ shows an excellent agreement between experiments and simulation results. The last transition time $\tau_{G}$ can be easily obtained by equating Eqs. (6) and (7), that is,

$$
\frac{D_{0} \tau_{G}}{\sigma^{2}}=\frac{N}{6}\left(\pi R^{*}\right)^{2} .
$$
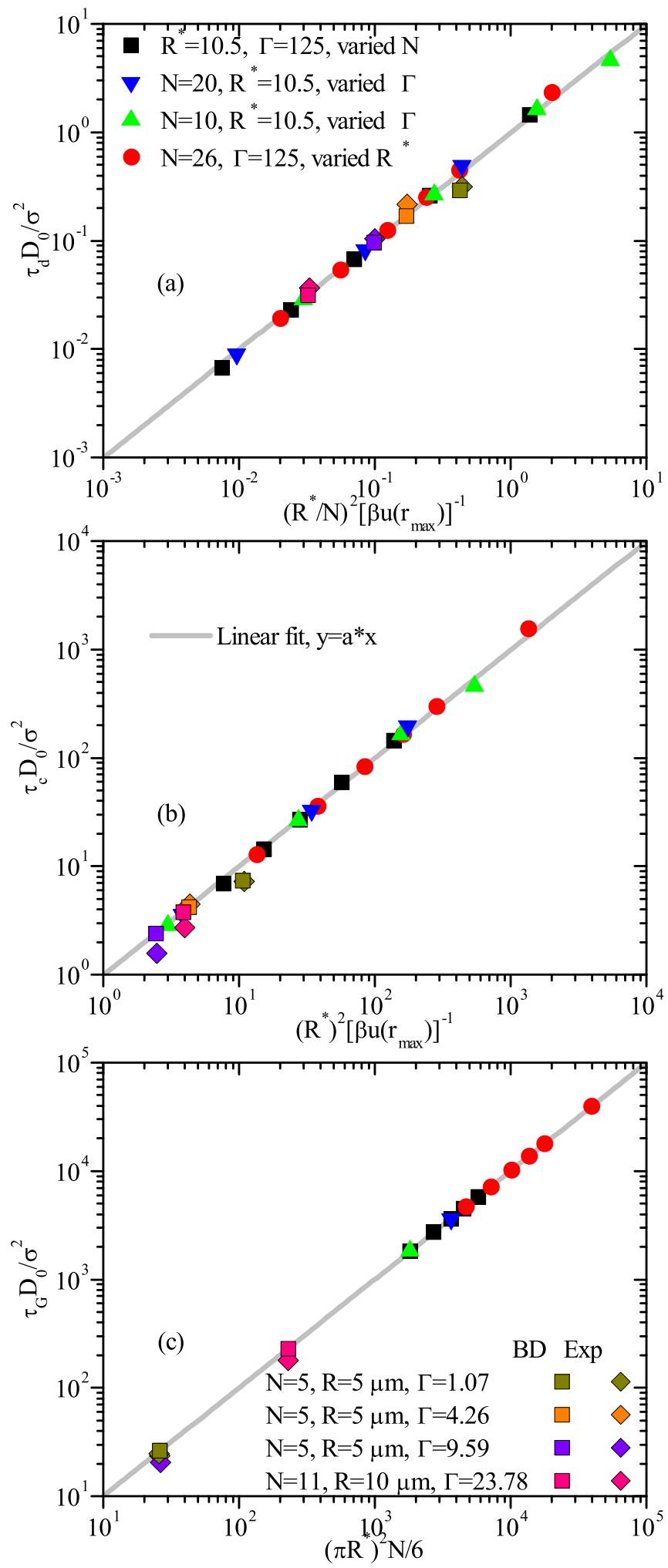

FIG. 5. Transition times $\tau_{d}, \tau_{c}$, and $\tau_{G}$ for the crossover between the different regimes for paramagnetic colloids diffusing along a circle of radius $R$. In (a) and (b), the straight line is the linear fit with slope $a \approx 0.99 \pm 1.74 \times 10^{-2}$. The line in (c) is the best linear fit with slope $a \approx 1.0 \pm 2.78 \times 10^{-14}$. Both experimental and simulation results displayed in Figs. 3 and 4 are included. 


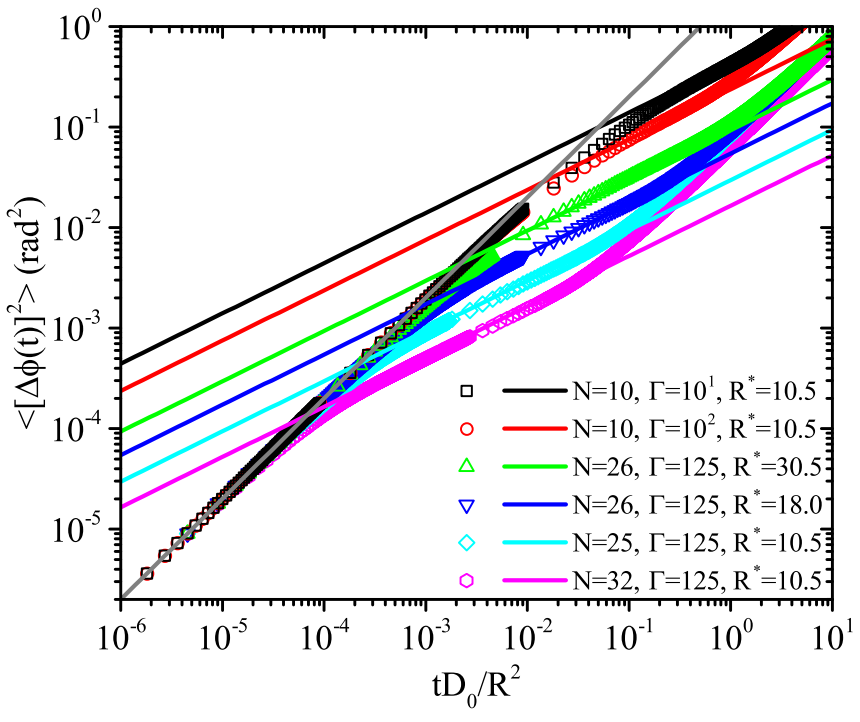

FIG. 6. Mean-square angular displacement for six paramagnetic colloidal systems obtained with the Ermak-McCammon (EM) algorithm in Eq. (2). Bold lines represent Eq. (5) with the mobility factor given by Eq. (11) evaluated using the physical parameters as indicated.

Figure 5(c) shows clearly that the previous expression describes remarkably well both experimental data and simulation results. We obtained the experimental and simulation points in Fig. 5 as follows: their vertical values from the crosses between the respective linear fits (on a log-log scale) of the different temporal regimes in Figs. 3 and 4 and their horizontal values using the position of the first maxima of the angular distribution functions shown in Fig. 2 in the expression for the interaction potential.

\section{ASYMPTOTIC BEHAVIOR}

While we show the expression for the SF mobility in Eq. (11), another immediate result is the availability of writing the subdiffusive regime as a function of the first transition time:

$$
\left\langle[\Delta \phi(t)]^{2}\right\rangle=\frac{F}{R^{2}} \sqrt{t}=2 \frac{D_{0}}{R^{2}} \sqrt{\tau_{d} t} .
$$

By doing this, we notice that Eq. (14) is like the diffusive behaviors described in Eqs. (4) and (6), which give us the possibility to construct a relationship that connects all the different time regimes in terms of the crossover times: the first diffusive regime $\left(\left\langle[\Delta \phi(t)]^{2}\right\rangle \sim t\right)$, the subdiffusive regime $\left(\left\langle[\Delta \phi(t)]^{2}\right\rangle \sim \sqrt{t}\right)$, the second diffusive regime $\left(\left\langle[\Delta \phi(t)]^{2}\right\rangle \sim t / N\right)$, and the geometrical regime $\left(\left\langle[\Delta \phi(t)]^{2}\right\rangle=\pi^{2} / 3\right)$. To this end, we first propose an equation that describes both the first diffusive and the subdiffusive regimes given by Eqs. (4) and (14), respectively. We then use an exponential decay associated with the crossover time $\tau_{d}$ :

$$
\left\langle[\Delta \phi(t)]^{2}\right\rangle=2 \frac{D_{0}}{R^{2}} t\left[1-\exp \left(-\frac{\tau_{d}}{t}\right)\right]^{1 / 2} .
$$

This equation indeed displays the diffusive behavior at short times, while at longer times, it will indefinitely describe the subdiffusive one, recovering both time regimes.

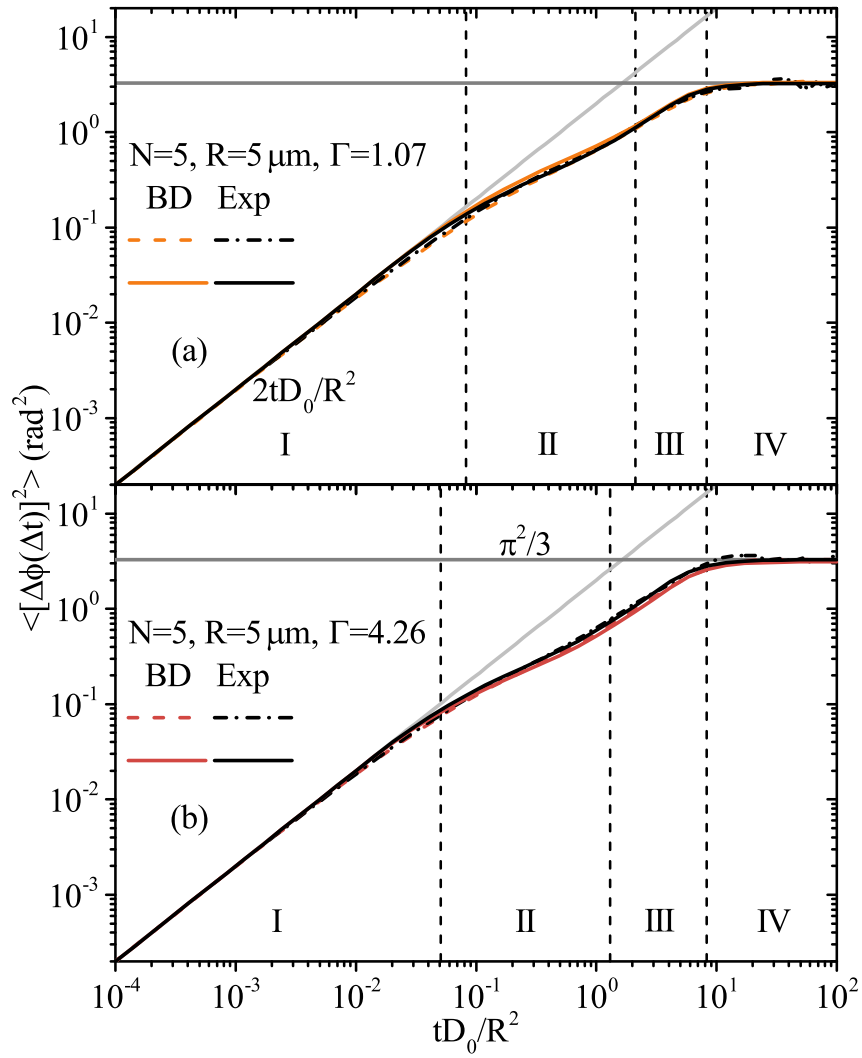

FIG. 7. Comparison of the mean-square angular displacement for the systems displayed in Figs. 3(a) and 3(b) (dotted lines, orange and red for Brownian dynamics (BD) and black for experiments) and Eq. (17) (continuous lines with the same color code) using the crossover times reported in Fig. 5.

Analogously, for the second transition, we now propose another exponential decay, but in this case, the transition is from $\sqrt{t}$ to $t$ linked to the crossover time $\tau_{c}$ :

$$
\left\langle[\Delta \phi(t)]^{2}\right\rangle=2 \frac{D_{0}}{R^{2}} t\left[\frac{1-\exp \left(-\frac{\tau_{d}}{t}\right)}{1-\exp \left(-\frac{\tau_{c}}{t}\right)}\right]^{1 / 2} .
$$

If the angle was not bound, this equation would describe correctly the MSAD (data not shown). However, we have used the bounds convention, i.e., $\phi \in[0, \pi]$. With this condition, the last transition time occurs when the MSAD reaches a plateau, changing the linear dependence with time to a constant value. This dynamical feature can be included in Eq. (16) by considering a term that recovers the geometrical time regime as follows:

$$
\begin{aligned}
\left\langle[\Delta \phi(t)]^{2}\right\rangle= & 2 \frac{D_{0}}{R^{2}} t\left[\frac{1-\exp \left(-\frac{\tau_{d}}{t}\right)}{1-\exp \left(-\frac{\tau_{c}}{t}\right)}\right]^{1 / 2} \\
& \times\left[1-\exp \left(-\frac{\tau_{G}^{2}}{t^{2}}\right)\right]^{1 / 2} .
\end{aligned}
$$

To test the accuracy of this equation, the crossover times reported in Fig. 5 were used in such an expression, and the resulting MSAD was compared with its corresponding counterpart. In all cases, the agreement is rather good. For 
illustrative purposes, in Fig. 7, we only show a comparison between the results reported in Figs. 3(a) and 3(b) and Eq. (17).

\section{CONCLUDING REMARKS}

In this paper, we have studied the dynamical behavior of paramagnetic particles confined to move along a circle. Although this system was already studied in Refs. $[9,10]$, we demonstrated here features in the particle dynamics when the circle geometry is considered; those features are also corroborated with experiments performed at very long times that clearly reached the predicted geometrical time regime. This paper highlights that small and closed systems exhibit a richer dynamical behavior than the standard scenario of SFD along a straight and infinite line, where the MSD behaves, at long times, as $\sim \sqrt{t}$.

Furthermore, we provided evidence on a firm ground of the existence of four temporal regimes of the stochastic dynamics of a tagged particle confined along a circle. Although these regimes have been previously studied only numerically [4], here, we showed a complete picture of the phenomenon, which includes experiments, simulations, and a predictive analytical theory. These regimes are described in terms of the MSAD, where good consistency was found between the experimental measurement and the corresponding theoretical prediction. The first and second temporal regimes correspond to the usual transition from free-particle diffusion to the SF behavior, respectively, since the particles cannot pass through each other. However, after the SF regime, a new transition to a diffusion type regime emerged with a reduced diffusion coefficient given by $D_{0} / N$. This phenomenon, not observed in previous experiments $[9,10]$, is an additional feature of the supported manifold $S^{1}$ since the compactness of the circle implies the finite size of the system, as well as a collective state where all particles behave as one ring-shaped particle with a rotational Brownian motion. After this stage, the geometrical regime appeared as a consequence of the finite length of the circle.

Our results also allowed us to estimate expressions for the three transition times between the temporal regimes, and an expression for the SF mobility factor $F$. The first transition time turned out to be inversely proportional to the square value of the density, whereas the second transition time is proportional to the radius square of the circle. However, both transition times are inversely proportional to the value of the interaction potential divided by the thermal energy $\beta u\left(r_{\max }\right)$, where $r_{\max }$ corresponds to the position of the first peak of the angular distribution function. In addition to that, we found that the third transition time is independent of the interaction potential and temperature and becomes proportional to the square of the number of particles in the colloidal system. Regarding the analytical structure of the transition times and the behavior of the MSAD, we deduced the SF mobility $F$. In this case, we found that $F$ is inversely proportional to the product between the particle density and the square root of $\beta u\left(r_{\max }\right)$. In contrast to other expressions for the mobility coefficient (for instance, the one in Ref. [8]), our expression has a direct connection with the interaction potential, which can also be used to probe directly the interaction between the particles. We also provided an accurate analytical expression that can easily reproduce either experiments or simulations in terms of the transition times.

Our approach can be extended in various directions. For instance, the EM algorithm implemented on the circle can be used for other types of interaction potentials. In addition, the full formulation of the EM algorithm on curved manifolds can be used to explore the behavior of the dynamics of a tracer particle in different geometries, where curvatureinduced inhomogeneities are relevant. Recently, there has been an important interest in the study of active particle systems since they appear in a broad range of contexts; the SFD of active colloids in confined geometries [26-28] could be modeled with our approach including the active internal degree of freedom in our EM algorithm on curved manifolds. Furthermore, by trapping paramagnetic particles along a closed curve using optical tweezers, controlling the temperature using a second laser beam [29], and allowing changes from the external magnetic field as a thermodynamic variable, it might be possible to build and design colloidal heat engines with a few bodies [30], where our approach could be essential. Finally, we could extend our findings by introducing the number density $\rho=N / 2 \pi R$, allowing us to rewrite the transition times and the SF mobility in a more general description.

\section{ACKNOWLEDGMENTS}

A.V.-B. acknowledges the financial support provided by Conacyt (No. CVU 417675). Further financial support by Conacyt (Grants No. 237425 and No. 287067, and Red Temática de la Materia Condensada Blanda) is gratefully acknowledged. The authors also thank the General Coordination of Information and Communications Technologies at Cinvestav for providing high-performance computing resources on the Hybrid Cluster Supercomputer "Xiuhcoatl," which have contributed to the research results reported in this paper. R.C.-P. also acknowledges the financial support provided by the Marcos Moshinsky fellowship 2013-2014. A.O.-A. and P.T. acknowledge support from the European Research Council under Grant Agreement No. 811234.

\section{APPENDIX: FIRST TRANSITION TIME $\tau_{d}$ FROM PERTURBATION ANALYSIS}

Here, we provide a perturbation analysis to determine an expression for the first transition time $\tau_{d}$. The key observation is that, for a large value of the amplitude $\Gamma$ and a large value of the number of particles $N$, the value for the first peak of the angular distribution function is approximately $\phi_{\max } \approx \frac{2 \pi}{N}$. This feature allows us to introduce the hypothesis that, in this situation, the particles in the system are localized around the mechanical equilibrium positions with angles $\phi_{i}^{\mathrm{eq}}=\frac{2 \pi}{N}(i-$ 1) for $i=1, \ldots, N$. In addition, it is not difficult to show that the deterministic term $g_{i}:=\beta D_{0} \mathbf{F}_{i} \cdot \mathbf{T}_{i} \tau$ of the EM algorithm [Eq. (2)] is given by

$$
g_{i}\left(\phi_{i j}\right)=\sigma\left(\frac{\tau}{\tau_{\Gamma}}\right) \sum_{j \neq i}^{N} \frac{\cos \left(\frac{\phi_{i j}}{2}\right) \sin \left(\frac{\phi_{i j}}{2}\right)}{\left|\sin \left(\frac{\phi_{i j}}{2}\right)\right|^{5}},
$$


where $\tau_{\Gamma}=16 R^{4} /\left(3 \sigma^{2} D_{0} \Gamma\right)$. It is clear that the function $g_{i}$ becomes zero at equilibrium positions since the forces between all the particles cancel out. Now we carry out the perturbation $\phi_{i j}=\phi_{i j}^{\mathrm{eq}}+\eta_{i j}$, where $\phi_{i j}^{\mathrm{eq}}=\frac{2 \pi}{N}(i-j)$, and $\eta_{i j}$ is a fluctuation around the equilibrium configuration. The expansion of $g_{i}$ in the first order of $\eta_{i j}$ is

$$
g_{i}\left(\phi_{i j}\right) \approx-\left(\frac{\tau}{2 R^{*} \tau_{\Gamma}}\right) \sum_{j \neq i}^{N} \mathcal{P}_{i j} \eta_{i j}
$$

where the constants $\mathcal{P}_{i j}$ are given by $\mathcal{P}_{i j}=[1+$ $\left.3 \cos ^{2}\left(\phi_{i j}^{\mathrm{eq}} / 2\right)\right] /\left|\sin \left(\phi_{i j}^{\mathrm{eq}} / 2\right)\right|^{5}$. The fluctuation can be decomposed as $\eta_{i j}=\eta_{i}-\eta_{j}$, with $\eta_{i}=\delta \mathbf{r}(\tau) \cdot \mathbf{T}_{i}\left(\phi_{\mathrm{i}}^{\mathrm{eq}}\right)$ satisfying the fluctuation-dissipation theorem $\left\langle\eta_{i} \eta_{j}\right\rangle=2 \delta_{i j} D_{0} \tau$.

Next, we compute the first term of Eq. (8), equivalent to $\left\langle g_{i}^{2}\right\rangle$, using $\left\langle\eta_{i j} \eta_{i k}\right\rangle=2 D_{0} \tau\left(1+\delta_{j k}\right)$ for $i \neq j$ and $i \neq k$. Then

$$
\left\langle g_{i}^{2}\right\rangle=2 D_{0} \tau\left(\frac{\tau}{2 R^{*} \tau_{\Gamma}}\right)^{2}\left[\left(\sum_{j \neq i} \mathcal{P}_{i j}\right)^{2}+\sum_{j \neq i} \mathcal{P}_{i j}^{2}\right] .
$$

For simplicity, let us take $i=1$ for the tagged particle, and $j=2$ and $j=N$ for the first neighboring particles. Thus, for large $N$, one has $\left(\sum_{j} \mathcal{P}_{i j}\right)^{2}+\sum_{j} \mathcal{P}_{i j}^{2} \simeq 96 N^{10} / \pi^{10}$. Using this approximation, the MSAD turns out to be

$$
\left\langle\left[\Delta s_{i}(\tau)\right]^{2}\right\rangle \approx 2 D_{0} \tau\left[1+24\left(\frac{\tau N^{5}}{R^{*} \tau_{\Gamma} \pi^{5}}\right)^{2}\right] .
$$

Now we choose for the interaction amplitude the value $\Gamma=\beta u\left(r_{\max }\right)\left(r_{\max }^{*}\right)^{3}$, where approximately $r_{\max }^{*}=2 \pi R^{*} / N$ according to the observation performed at the beginning of the Appendix. The first transition time occurs when the second term inside the square parenthesis in Eq. (A4) is $\sim 1$. Therefore, one finds

$$
\frac{D_{0} \tau_{d}}{\sigma^{2}}=\frac{2 \pi^{2}}{3 \sqrt{24}}\left(\frac{R^{*}}{N}\right)^{2}\left[\beta u\left(r_{\max }\right)\right]^{-1} .
$$

The contribution of the next neighbors does not change the value $\mathcal{C}_{\text {th }}=2 \pi^{2} /(3 \sqrt{24}) \approx 1.3$ significantly. Notwithstanding, the factor $\mathcal{C}_{\text {th }}$ obtained with the perturbation theory differs slightly from the simulation result, shown in Fig. 5, the transition time has the correct structural dependence $\left(\frac{R^{*}}{N}\right)^{2}\left[\beta u\left(r_{\max }\right)\right]^{-1}$.
[1] M. D. Graham, Microhydrodynamics, Brownian Motion, and Complex Fluids, Vol. 58 (Cambridge University Press, Cambridge, 2018).

[2] G. Nägele, On the dynamics and structure of charge-stabilized suspensions, Phys. Rep. 272, 215 (1996).

[3] J. K. G. Dhont, An Introduction to Dynamics of Colloids (Elsevier, Amsterdam, 1996).

[4] P. Castro-Villarreal, A. Villada-Balbuena, J. M. MéndezAlcaraz, R. Castañeda-Priego, and S. Estrada-Jiménez, A Brownian dynamics algorithm for colloids in curved manifolds, J. Chem. Phys. 140, 214115 (2014).

[5] O. A. Ramírez-Garza, J. M. Méndez-Alcaraz, and P. GonzálezMozuelos, Structural and dynamic inhomogeneities induced by curvature gradients in elliptic colloidal halos of paramagnetic particles, J. Chem. Phys. 146, 194903 (2017).

[6] R. N. Zia and J. F. Brady, Theoretical microrheology, in Complex Fluids in Biological Systems: Experiment, Theory, and Computation, edited by S. E. Spagnolie (Springer New York, New York, 2015), pp. 113-157.

[7] A. Taloni, O. Flomenbom, R. Castañeda-Priego, and F. Marchesoni, Single file dynamics in soft materials, Soft Matter 13, 1096 (2017).

[8] M. Kollmann, Single-File Diffusion of Atomic and Colloidal Systems: Asymptotic Laws, Phys. Rev. Lett. 90, 180602 (2003).

[9] Q.-H. Wei, C. Bechinger, and P. Leiderer, Single-file diffusion of colloids in one-dimensional channels, Science 287, 625 (2000).

[10] C. Lutz, M. Kollmann, and C. Bechinger, Single-File Diffusion of Colloids in One-Dimensional Channels, Phys. Rev. Lett. 93, 026001 (2004).

[11] G. M. Schütz, Exact solution of the master equation for the asymmetric exclusion process, J. Stat. Phys. 88, 427 (1997).

[12] L. Lizana and T. Ambjörnsson, Single-File Diffusion in a Box, Phys. Rev. Lett. 100, 200601 (2008).
[13] J.-B. Delfau, C. Coste, and M. Saint Jean, Single-file diffusion of particles in a box: Transient behaviors, Phys. Rev. E 85 , 061111 (2012).

[14] J.-B. Delfau, C. Coste, and M. Saint Jean, Single-file diffusion of particles with long-range interactions: Damping and finitesize effects, Phys. Rev. E 84, 011101 (2011).

[15] G. Tarjus, F. Sausset, and P. Viot, Statistical mechanics of liquids and fluids in curved space, Adv. Chem. Phys. 148, 251 (2011).

[16] T. Bohlein, J. Mikhael, and C. Bechinger, Observation of kinks and antikinks in colloidal monolayers driven across ordered surfaces, Nat. Mater. 11, 126 (2012).

[17] U. Siems and P. Nielaba, Transport and diffusion properties of interacting colloidal particles in two-dimensional microchannels with a periodic potential, Phys. Rev. E 91, 022313 (2015).

[18] P. Castro-Villarreal, Brownian motion meets Riemann curvature, J. Stat. Mech. (2010) P08006.

[19] A. Ortiz-Ambriz and P. Tierno, Engineering of frustration in colloidal artificial ices realized on microfeatured grooved lattices, Nat. Commun. 7, 10575 (2016).

[20] A. Oritz-Ambriz, aortiza/AODControls: Clean structure, https://zenodo.org/record/4013935 (2020).

[21] J. C. Crocker and D. G. Grier, Methods of digital video microscopy for colloidal studies, J. Colloid Interface Sci. 179, 298 (1996).

[22] K. Zahn, J. M. Méndez-Alcaraz, and G. Maret, Hydrodynamic Interactions May Enhance the Self-Diffusion of Colloidal Particles, Phys. Rev. Lett. 79, 175 (1997).

[23] P. Viveros-Méndez, J. Méndez-Alcaraz, and P. GonzálezMozuelos, Two-body correlations among particles confined to a spherical surface: Packing effects, J. Chem. Phys. 128, 014701 (2008).

[24] P. Viveros-Méndez, J. Méndez-Alcaraz, and P. GonzálezMozuelos, Formation and structure of colloidal halos in 
two-dimensional suspensions of paramagnetic particles, J. Chem. Phys. 136, 164902 (2012).

[25] A. Villada-Balbuena, Escalas temporales y efectos geométricos en sistemas brownianos, Ph.D. thesis, Cinvestav, Mexico City, 2018.

[26] S. J. Ebbens, Active colloids: Progress and challenges towards realising autonomous applications, Curr. Opin. Colloid Interface Sci. 21, 14 (2016).

[27] L. Zhang, Z. Xiao, X. Chen, J. Chen, and W. Wang, Confined $1 \mathrm{D}$ propulsion of metallodielectric janus micromotors on micro- electrodes under alternating current electric fields, ACS Nano 13, 8842 (2019).

[28] P. Dolai, A. Das, A. Kundu, C. Dasgupta, A. Dhar, and K. V. Kumar, Universal scaling in active single-file dynamics, Soft Matter 16, 7077 (2020).

[29] V. Blickle and C. Bechinger, Realization of a micrometre-sized stochastic heat engine, Nat. Phys. 8, 143 (2012).

[30] I. A. Martínez, É. Roldán, L. Dinis, and R. A. Rica, Colloidal heat engines: A review, Soft Matter 13, 22 (2017). 